\title{
Polarization in impression formation as a function of affect and range dispersion*
}

\author{
L. P. RICHARDSON \\ Montgomery County Community College, Blue Bell, Pa. 19422
}

The averaging model of impression formation was used in tests of the polarization effects produced by affect and range dispersion. A discrepancy score (departure from averaging) and an evaluative score were used in an impression formation task with trait adjectives. Support was found for two hypotheses which predicated that each factor would produce polarization in impression judgments. The results indicated that increases in range dispersion and negative affect produced polarization from the averaging model's predictions. The results also suggested that minor polarization may occur with positive affect traits that are homogeneous.

Numerous studies in the impression formation area have used Anderson's (1962) averaging model to account for their findings. While the model has not been successful in every test, it has demonstrated its effectiveness. The present study sought to examine two factors that have been suggested as threats to the model's predictions. Whether the effects of these factors are systematic and orderly enough to require a final modification of the model's functioning is not known. But there is evidence that these factors may produce polarization in evaluative judgments and, hence, a deviation from the simple averaging formulation.

The first factor is affect. It involves the question of whether the model functions as effectively with negative trait values as it does with positive ones. Early tests (Anderson, 1965; Anderson \& Jacobson, 1965) found the model to be effective with positive affect values but not with negative trait values. A recent study (Hamilton \& Huffman, 1971) has supported this. Anderson (1968a) had proposed that an additional parameter be added to the averaging model to account for the negative affert factor. The second factor presumed to cause polarization in the evaluative impression judgment is the range of dispersion or likableness in the stimulus values which are to be pooled and judged. Wyer \& Watson (1969) found this factor contributed significantly to polarization in the impression formation process.

The present study sought to examine these two factors and the extent of polarization from averaging model predictions that were associated with each factor. The present study relates to previous studies at three points. First, the confounding effects noted by Wyer \& Watson (1969) in testing the range dispersion factor have been controlled for in the present study. Second,

*This article is based upon data contained in a doctoral dissertation submitted to Temple University in partial fulfillment of requirements for the Ed.D. degree. Calvin F. Nodine sponsors the paper and takes full editorial responsibility for its contents. previous studies have used broad subranges in affect (e.g., $\mathrm{H}+, \mathrm{H}, \mathrm{M}+, \mathrm{M}$, etc.) from which normative values for trait set construction were obtained. The present study pursued a more precise and balanced construction procedure. Finally, in addition to the usual like-dislike or evaluative score, a discrepancy score was used in the present study to reflect the magnitude of deviation or polarization from the averaging model. This score was the absolute difference between the obtained impression value for a particular set of traits and the value that the averaging model predicted for that set.

Two predictions were made with respect to the affect and range dispersion factors. First, it was hypothesized that affect would produce significant differences in discrepancy scores. Specifically, it was predicted that discrepancy scores would increase as affect values changed from positive to neutral to negative. Second, it was hypothesized that the range dispersion factor would produce significant differences in both the evaluative or like-dislike and the discrepancy scores. The evaluative scores were expected to become smaller or negative as the size of the range dispersion increased. Finally, it was predicted that discrepancy scores would increase with increases in levels of the range dispersion factor.

\section{PROCEDURE}

Ss for this study were 102 undergraduate females in an introductory educational psychology course. From Anderson's (1968b) original list, adjectives with small standard deviations were selected and normative values obtained from participants 4 weeks prior to the study. From this original trait adjective pool, 27 traits were incorporated in nine experimental sets composed of three traits each. These sets were combined to represent levels of affect-negative, neutral, and positive-and three levels of range dispersion-low, medium, and high. The balancing of trait values to establish both of these factors can be seen in Table 1 . The mean affect value, as predicted by the averaging model, is indicated in the left column. The individual trait value combinations are in the body of the table.

The nine experimental sets were assembled into booklet form, with one triadic set per page. Also, on each page was a scale from 1 to 7 that had dislike, neutral, and like under the 1,4 , and 7 categories. A table of random numbers was consulted for ordering the pages in the booklet. To insure use of both ends of the scale, four biased sets were placed at the beginning of each booklet. These warm-up sets were not included in the data analysis. The instructions encouraged participants to give equal importance and careful attention to each trait and not to worry about traits which appeared to be contradictory to each other. After a preliminary analysis that is recommended (Winer, 1962) to test assumptions underlying analyses of variance with a repeated-measures design, the data were analyzed as a 3 by 3 factorial design through the use of the BMD $08 \mathrm{~V}$ program in the biomedical series (Dixon, 1970).

\section{RESULTS AND DISCUSSION}

The prediction relating to the affect factor was supported by the discrepancy scores. These means 
Table 1

Trait Value Combinations According to Affect and Range Dispersion

\begin{tabular}{llcc}
\hline \multirow{2}{*}{$\begin{array}{l}\text { Mean Affec- } \\
\text { tive Value } \\
\text { of Set }\end{array}$} & \multicolumn{3}{c}{ Range of } \\
\cline { 2 - 4 } & Low & Medium & High \\
\hline 5 (Positive) & $5-5-5$ & $4-5-6$ & $3-5-7$ \\
4 (Neutral) & $4-4-4$ & $3-4-5$ & $2-4-6$ \\
3 (Negative) & $3-3-3$ & $2-3-4$ & $1-3-5$ \\
\hline
\end{tabular}

increased from .95 to 1.04 to 1.21 as affect values shifted from positive (mean value of 5) to neutral (mean value of 4) to negative (mean value of 3 ). The $F$ value for these comparisons was $8.08(\mathrm{df}=2 / 198, \mathrm{p}<.01)$. A post hoc analysis indicated that of the three comparisons two were significant. That is, negative trait set discrepancy scores were significantly larger than positive or neutral trait set discrepancy scores. These results would seem to parallel earlier studies concerned with affect and the recent conclusion of Hamilton \& Huffman (1971) that greater weight is given to negative information than is given to positive.

The two predictions relating to the range dispersion factor were likewise supported by the two dependent measures. The evaluative scores decreased from 4.02 to 3.83 to 3.38 for the low, medium, and high range dispersion levels (see Table 1 for size of dispersion). The $F$ value $(F=24.74, d f=2 / 198, p<.01)$ and post hoc comparisons indicated that each of these increases was significant.

The discrepancy scores gave weaker support $(\mathrm{F}=3.25, \mathrm{df}=2 / 198, \mathrm{p}<.05)$ to the range dispersion hypotheses. Discrepancy scores were expected to increase with increases in the range dispersion level. But this linear expectation did not occur. Instead, a curvilinear pattern emerged with low and high range dispersion, producing larger discrepancy scores than the medium level, i.e., $1.10,1.12$, and .98 , respectively. This outcome was produced by two polarization patterns resulting in a significant interaction effect between affect and range dispersion, a finding similar to that noted in Wyer \& Watson (1969).

One of the polarization patterns was expected; the other was not. The expected pattern occurred with negative set values, i.e., a steady increase in the discrepancy scores occurred across the range dispersion factor with increases in negative affect values. This linear relationship between affect and polarization across the range dispersion factor was analogous to the findings reported by Wyer \& Watson (1969). These means were $.98,1.20$, and 1.50 for the low, medium, and high range dispersion levels, respectively.

The unexpected polarization pattern occurred with positive affect trait values. The direction of this pattern was contrary to that of the negative affect values. That is, as the positive traits of the stimulus person became less heterogeneous and more homogeneous (low range dispersion), there was a tendency to evaluate that person more positively than the averaging model predicted. Whether this phenomenon was a result of a particular trait or a characteristic of the sample as observed by others (Anderson, 1968a) will need further testing.

In summary, this study offered support for both factors as important elements in the impression formation process. The findings with the range dispersion factor would seem to support an analysis which gives heavier weighting to extreme values (Manis, Gleason, \& Dawes, 1966; Wyer \& Watson, 1969). The results of tests with affect were consonant with other studies dealing with this question. The reason or reasons why negative information is more crucial for impression judgment have yet to be defined.

\section{REFERENCES}

Anderson, N. H. Application of an additive model to impression formation. Science, $1962,138,817-818$.

Anderson, N. H. Averaging versus adding as a stimulus-combination rule in impression formation. Journal of Experimental Psychology, 1965, 70, 394-400.

Anderson, N. H. Application of a linear-serial model to a personality-impression task using serial presentation. Journal of Personality \& Social Psychology, 1968a, 10, 354-362.

Anderson, N. H. Likableness ratings of 555 personality-trait words. Journal of Personality \& Social Psychology, 1.968b, 9, 272-279.

Anderson, N. H., \& Jacobson, A. Effects of stimulus inconsistency and discounting instructions in personality impression formation. Journal of Personality \& Socia Psychology, 1965, 2, 531-539.

Dixon, W. J. (Ed.), Biomedical computer programs. Berkeley: University of California Press, 1970.

Hamilton, D. L., \& Huffman, L. J. Generality of impression-formation processes for evaluative and nonevaluative judgments. Journal of Personality \& Socia Psychology, 1971, 20, 200-207.

Manis, M., Gleason, T. C., \& Dawes, R. M. The evaluation of complex social stimuli. Journal of Personality \& Social Psychology, 1966, 3, 404-419.

Winer, B. J. Statistical principles in experimental design. New York: McGraw-Hill, 1962.

Wyer, R. S., \& Watson, S. F. Context effects in impression formation. Journal of Personality \& Social Psychology, 1969 12, 22-23.

(Received for publication February 26, 1973.) 